\title{
Some Monte Carlo Evidence for Adaptive Estimation of Unit-Time Varying Heteroscedastic Panel Data Models
}

\author{
G. R. Pasha \\ Department of Statistics, Bahauddin Zakariya University Multan, Pakistan \\ E-mail: drpasha@bzu.edu.pk \\ Muhammad Aslam \\ Department of Statistics, Bahauddin Zakariya University Multan, Pakistan \\ E-mail: aslamasadi@bzu.edu.pk
}

\begin{abstract}
In this paper, we present an adaptive estimator for panel data model with unknown unit-time varying heteroscedastic error component of unknown form by using probability weighted moments rather than conventional kernel estimators already available in the literature and then evaluate the finite sample performance of the proposed estimator in terms of efficiency and testing of hypothesis. The Monte Carlo evidence suggests that the proposed estimator performs adequately under different data generated processes, especially for small samples that are the most practical situations.
\end{abstract}

Keyword: Adaptive estimator, Probability weighted moments, Heteroscedasticity, Unit-time varying error component

\section{Introduction}

An extensive literature is available on the issue of heteroscedasticity in the context of cross-section and time-series data and to deal this problem, especially, for heteroscedasticity of unknown form, there are many adaptive results using nonparametric methods; see Carroll (1982), Delgado (1992), Hidalgo (1992), Robinson (1987), among many others. But for panel data, referred as cross-section data sampled over different time period for the same economic agent, the issue of heteroscedasticity has not been studied as much extensively. In recent literature, only few references can be found so for; see Roy (1999), Baltagi (1998), Li and Stengos (1994), Baltagi and Griffin (1988), Randolph (1988), Mazodier and Trongnon (1978), etc. Randolph's work is based on unbalanced panel data while Baltagi and Griffin (1988) extend on Mazodier and Trongnon's work for the balanced case. Baltagi and Griffin (1988) consider heteroscedasticity coming in through the unit-specific error. They simply use an empirical example to provide some support for their estimators. Also the procedure proposed by them requires a large time component for the panel, which may not always be available. Li and Stengos (1994) provide an adaptive estimator for panel data models with unit-time varying heteroscedastic error component. Roy (1999) proposes an adaptive estimator for the estimation of panel models with unit-specific heteroscedastic error component by giving some Monte Carlo evidence to support the 
proposal and unlike the estimator proposed by Baltagi and Griffin (1988), there is no need for large time component.

In this paper, an adaptive procedure is formulated for the estimation of panel data model with unit-time varying heteroscedastic error component of unknown form by replacing the kernel estimator, presented by Li and Stengos (1994), with probability weighted moments, explained by Downton (1966) and Greenwood (1979) due to their robustness over conventional moments for more efficient inferences. Monte Carlo studies on the lines of Rilstone (1991), $\mathrm{Li}$ and Stengos (1994) and Roy (1999) are carried out to evaluate the performance of the estimator. Since $\mathrm{Li}$ and Stengos (1994) find the proposed estimator to perform adequately comparing it with different standard estimators so, in this paper, we just compare the new proposed estimator with the estimator given by $\mathrm{Li}$ and Stengos (1994). In section 2, we give unit-time varying heteroscedastic error component panel data model. In section 3 , an adaptive estimator is proposed on the basis of probability weighted moments. In section 4, Monte Carlo experiment is carried out under two different data generating procedures to rank the performance of the adaptive estimator. In section 5, results and discussion are presented and also performance of the estimators is evaluated in testing of hypotheses. Finally, section 6 concludes.

\section{Unit-time Varying Heteroscedastic Error Component Panel Data Model}

A standard error component model, discussed by Hsiao (1985), Li and Stengos (1994), Baltagi (1995), and Roy (1999) among others is given as

$$
y_{i t}=x_{i t} \beta+\mu_{i}+v_{i t}
$$

where $i=1,2, \ldots, N ; t=1,2, \ldots, T, x_{i t}$ is $1 \times k, \mu_{i}$ is the unit-specific error component with $\mu_{i t} \sim$ i.i.d. $\left(0, \sigma_{\mu}^{2}\right)$ while $v_{i t}$ is the unit-time varying error component and $\sim$ i.i.d. $\left(0, \pi_{i j}\right)$. Where $\pi_{i j}=\pi\left(x_{i j}\right)=\operatorname{Var}\left(v_{i t}\right)$ and shows that unit-time varying error component is heteroscedastic.

The vector-matrix form of (2.1) can be

$$
y=x \beta+Z \mu+v
$$

where $Z=I_{N} \otimes e_{T}, e_{T}$ is a $T$ dimensional column vector of ones and $\mu=\left[\mu_{1}\right.$, $\left.\mu_{2}, \ldots, \mu_{N}\right]^{\prime} . y$ and $v$ are $N T \times 1$ column vectors of the dependent variable and the unit-time varying error component, respectively while $x$ is an $N T \times K$ matrix of repressors.

The inverse of the conditional variance-covariance matrix of the error term in (2.2) denoted by $\Omega^{-1}$, following Baltagi and Griffin (1988) and Roy (1999), is given as

$$
\Omega^{-1}=\operatorname{diag}\left[1 / \sigma_{i}^{2}\right] \otimes\left(J_{T}{ }^{\prime} T\right)+\operatorname{diag}\left[1 / \sigma_{v}^{2}\right] \otimes\left(I_{T}-J_{T}{ }^{\prime} T\right)
$$

where $\sigma_{i}^{2}=T \omega_{i}+\sigma_{v}^{2} \forall i$ and $J_{T}$ is a square matrix of ones of dimension $T$. 
The true GLS estimator of $\beta$ is

$$
\widetilde{\beta}=\left(x^{\prime} \Omega^{-1} x\right)^{-1} x^{\prime} \Omega^{-1} y
$$

Since (2.4) involves working with a $N T \times N T\left(\Omega^{-1}\right)$ matrix which can be quite demanding if one has a large data set so (2.4) can be rewritten as

$$
\widetilde{\beta}=\left(\sum_{i=1}^{N} x_{i}^{\prime} B_{i}^{-1} x_{i}\right) \quad\left(\sum_{i=1}^{N} x_{i}^{\prime} B_{i}^{-1} y_{i}\right)
$$

where $x_{i}$ is a $T \times K$ matrix of repressors for the $i$-th individual, $y_{i}$ is $T \times 1$ and $B_{i}^{-1}$ is given as

$$
B_{i}^{-1}=\frac{1}{\gamma_{i}\left(1-\rho_{i}\right)}\left[I_{T}-\frac{e_{T} e_{T}^{\prime} \rho_{i}}{\left(1-\rho_{i}+T \rho_{i}\right)}\right]
$$

with $\rho_{i}=\sigma_{\mu}^{2} / \gamma_{i}$ and $\gamma_{i}=\sigma_{\mu}^{2}+\pi_{i j}$ (the total variance)

\section{Adaptive Estimator}

To obtain the Estimated Generalized Least Squares (EGLS) of $\beta$ (as in 2.5), we need to estimate $\rho_{i}$ and $\gamma_{i}$ given in (2.6). Following Hsiao (1986) and Li and Stengos (1994), $\sigma_{\mu}^{2}$ can be estimated as

$$
\hat{\sigma}_{\mu}^{2}=\frac{\sum_{i=1}^{N} \sum_{t=2}^{T} \hat{\mu}_{i t} \hat{\mu}_{i, t-1}}{N(T-1)}
$$

where $\hat{\mu}_{i t}$ is the OLS residual.

$\mathrm{Li}$ and Stengos (1994) and Roy (1999) propose a kernel estimator of $\gamma_{i}$ as

$$
\hat{\gamma}_{i}=\frac{\sum_{j=1}^{N} \sum_{t=1}^{T} \hat{\mu}_{j t}^{2} K\left(\frac{\bar{x}_{i}-x_{j t}}{h}\right)}{\sum_{j=1}^{N} \sum_{t=1}^{T} K\left(\frac{\bar{x}_{i}-x_{j t}}{h}\right)}
$$

where $K($.$) is the kernel function as K\left(\varphi_{i t}\right)=\frac{1}{\sqrt{2 \pi}} \exp \left(-\frac{\varphi_{i t}^{2}}{2}\right)$ with $h$ as the smoothing parameter. Then after obtaining the estimate of $\gamma_{i}$, the estimate of $\pi_{i j}$ can be obtained as $\hat{\pi}_{i t}=\hat{\gamma}_{i}-\hat{\sigma}_{\mu}^{2}$ and then after calculating $\hat{\rho}_{i}=\hat{\sigma}_{\mu}^{2} / \hat{\gamma}_{i}$, the estimate of $\beta(2.5)$ can be achieved.

But in this paper, we present an adaptive estimator in the presence of unittime varying heteroscedastic error component by estimating $\operatorname{Var}\left(v_{i t}\right)=\pi_{i j}$ using probability weighted moments discussed by Downton (1966) as linear estimate of the standard deviation of the normal distribution as

$$
S_{p w}=\frac{\sqrt{\pi}}{n} \sum_{i=1}^{n}\left[Y_{i}-2\left(1-\frac{i-0.5}{n}\right) Y_{i}\right]
$$


For our problem

$$
\hat{\pi}_{i t}=\left[\frac{\sqrt{\pi}}{i} \sum_{i=1}^{N}\left\{y_{(i t)}-2\left(1-\frac{i-0.5}{N}\right) y_{(i t)}\right\}^{2}\right]
$$

where $y_{(i t)}$ are the ordered observations and $(i-0.5) / N$ is the empirical distribution function $F_{N}(Y)$.

Now the adaptive estimator can be given by reformulating (2.5) as

$$
\hat{\beta}=\left(\sum_{i=1}^{N} x_{i}^{\prime} \hat{\tau}_{i}^{-1} x_{i}\right)^{-1}\left(\sum_{i=1}^{N} x_{i}^{\prime} \hat{\tau}_{i}^{-1} y_{i}\right)
$$

where

$$
\hat{\tau}_{i}^{-1}=\frac{1}{\hat{\gamma}_{i}\left(1-\hat{\rho}_{i}\right)}\left[I_{T}-\frac{e_{T} e_{T}^{\prime} \hat{\rho}_{i}}{\left(1-\hat{\rho}_{i}+T \hat{\rho}_{i}\right)}\right]
$$

\section{Monte Carlo Experiment}

In this section, Monte Carlo experiment is carried out to study the performance of the proposed estimator. For comparative purposes, the design of the Monte Carlo experiment is as same as used by Rilstone (1991), Li and Stengos (1994), and Roy (1999). The following model is considered:

$$
y_{i t}=\beta_{0}+\beta_{1} x_{i t}+\mu_{i}+v_{i t} ; \quad i=1,2, \ldots, N ; t=1,2, \ldots, T
$$

where $x_{i t}=0.5 \omega_{i, t-1}+\omega_{i t}$ and $\omega_{i t}$ is generated by two different data generating processing (DGP), namely

DGP-I: $\omega_{i t} \sim$ i.i.d. $U(0,2)$,

DGP-II: $\omega_{i t} \sim$ i.i.d. $e^{v_{i t}}$ and $v_{i t}$ i.i.d. $N\left(0,(0.4)^{2}\right)$ i.e., $\omega_{i t}$ is Lognormal.

The parameters $\beta_{0}$ and $\beta_{1}$ are assigned the values 5 and 0.5 , respectively and $\mu_{i}$ s are generated as $\mu_{i} \sim N\left(0, \sigma_{\mu}^{2}\right)$. We generate $v_{i t}$ as $v_{i t} \sim$ i.i.d. $N\left(0, \pi_{i j}\right)$. Where $\pi_{i j}=\operatorname{Var}\left(v_{i t}\right)=\pi\left(x_{i j}\right)$ shows that unit-time varying error component is heteroscedastic and $\pi\left(x_{i j}\right)=\alpha^{2}\left(1+\lambda x_{i t}\right)^{2}$. Let $\bar{\pi}=E(\pi(x))$, denotes the expected variance of $v_{i t}$. On the lines of Li and Stengos (1994), we fix the total variance $\gamma_{i}=\sigma_{\mu}^{2}+\bar{\pi}=8$ and define $\rho=\sigma_{\mu}^{2} /\left(\sigma_{\mu}^{2}+\bar{\pi}\right)$, where $\rho$ takes values, $0.2,0.5$, and 0.8 . For each fixed value of $\rho$, the value of $\sigma_{\mu}^{2}$ is calculated to vary the share of the variance of the unit-specific error term in the total variance. For each fixed value of $\rho$ and $\sigma_{\mu}^{2}, \lambda$ is assigned values $0,1,2$, and 3 where 0 denotes homoscedastic unit-time varying error while the degree of heteroscedasticity increases as the value of $\lambda$ becomes larger. For a fixed $\sigma_{\mu}^{2}$, a value of $\bar{\pi}$ is obtained (as $\bar{\pi}=8-\sigma_{\mu}^{2}$ ) using the different values of $\lambda(0,1$, 
2 , and 3) and the values of $\alpha$ are chosen in such a way that the expected variance $\bar{\pi}$ is fixed for different values of $\lambda$. Then the values of $\omega_{i}$ are obtained for each $\sigma_{\mu}^{2}$ under the four different $\lambda$ values. Following Roy (1999) and Li and Stengos (1994), the two schemes for sample sizes are used:

i) $\quad N=50, T=3$; i.e., $N \times T=150$

ii) $\quad N=100, T=3$; i.e., $N \times T=300$

For the estimation part, the estimator proposed by Li and Stengos (1994), is evaluated on the same directions of Li and Stengos (1994), using the normal kernel and smoothing parameter values $h(0.8,1,1.2)$ and this estimation is denoted as generalized least squares adaptive (GLSAD) estimation as named by $\mathrm{Li}$ and Stengos (1994). The proposed adaptive estimator (3.5) is determined and denoted as probability weighted generalized least squares (PWGLS).

Li and Stengos (1994) report the adequacy of the proposed GLSAD estimator by comparing it with different standard estimators i.e., OLS estimator, conventional GLS estimator of one-way error component model that assumes the remainder error term $v_{i t}$ is homoscedastic (GLSH), and WITHIN estimator. So in this section, the adaptive PWGLS estimator is just compared with the GLSAD estimator of Li and Stengos (1994) and the OLS estimator. For this purpose, the efficiency of PWGLS relatives to GLSAD and OLS is computed. The relative efficiency (R.E) is defined here as the ratio of the mean square of the estimator under consideration to the mean square error of PWGLS e.g., R.E = M.S.E (GLSAD)/M.S.E (PWGLS).

The efficiency of the estimators is not only concern while estimating the model (4.1) but performance of the hypothesis tests regarding the coefficients is also counted. To illustrate the impact of the both estimators on hypothesis testing, a test $\mathrm{H}_{0}: \beta_{1}=0.5$ against $\beta_{1} \neq 0.5$ is considered and the relevant $p$-values are computed. The larger $p$-value gives the statistical non-significance and indicates the stronger evidence for accepting the null hypothesis; $\beta_{1}=0.5$.

\section{Results and Discussions}

Table 1 gives the relative efficiency under DGP-I, with a sample size of 150 , formed with $\mathrm{N}=50, \mathrm{~T}=3$. It shows when $\lambda=0$ then for all the cases of $\rho=$ $0.2,0.5,0.8$, both GLSAD estimator and the proposed PWGLS estimator perform almost with equal efficiency. In other words, when the unit-time varying error term is homoscedastic then one may use any one from the both estimators. But for all different values of $\rho$, the OLS estimator performs poorly and bears relatively great efficiency loss for smaller values of $\sigma_{\mu}^{2}$ (larger contribution of $\bar{\pi}$ ). The OLS estimator performs worse since it ignores both the 
effects of $\mu_{i}$ and $v_{i t}$. When $\lambda=1$, the PWGLS estimator performs well for all the cases of $\rho=0.2,0.5,0.8$ but as the contribution of $\sigma_{\mu}^{2}$ increases in the total variance the relative efficiency of the PWGLS estimator begin to decrease. That is for the larger role of $\bar{\pi}$ in the total variance, the proposed estimator becomes more attractive. For the higher degree of heteroscedasticity $(\lambda=2,3)$, the PWGLS outperforms as compared to GLSAD and OLS. The sensitivity of the GLSAD estimator can be observed by the selection of the smoothing parameter $h$. Generally, for different values of $h$ the GLSAD estimator varies in performance that also verifies the findings of Roy (1999) and Li and Stengos (1994) no such problem is faced for the use of PWGLS.

Table 2 gives the relative efficiency under DGP-I, with a sample size of 300 , formed with $\mathrm{N}=100, \mathrm{~T}=3$. The results in this table almost possess the same qualitative interpretations as that is in Table 1. The PWGLS performs quite adequately in all the cases as discussed above. It can again be noted that with the increase in sample size from 150 to 300, the efficiency of the PWGLS decreases, although in small amount. For example, In Table 1, for the case of $\rho=0.2$ (and $\lambda=1,2,3$ ), the relative efficiency ranges from 1.0475 to 1.2514 that falls in 1.0067 to 1.2455 in Table 2. Similar decrease can be found in the cases when $\rho=0.5,0.8$. These results again signify the attractiveness of the PWGLS for small samples.

Table 3 and Table 4 show the relative efficiency under DGP-II where regressors are generated by lognormal distribution and the sample sizes are 150 and 300. The adaptive estimator PWGLS gives the results in the same fashion as under DGP-I. Efficiency benefits of the proposed estimator can be found for smaller values of $\rho$ and $\sigma_{\mu}^{2}$ (larger contribution of $\bar{\pi}$ ) almost in the same routine as discussed above.

The performance of the estimators in the hypothesis tests regarding the coefficients is also taken into account while estimating the model. Table 5 shows the $p$-values for testing the hypothesis $\mathrm{H}_{0}: \beta_{1}=0.5$ against $\beta_{1} \neq 0.5$ to see the impact of the both estimators, GLSAD and the proposed PWGLS under DGP-I with sample size 150. The larger $p$-value gives the statistical non-significance and indicates that there is no significant difference between the estimated value of slope coefficient and the parametric value 0.5 or, in other words, a stronger evidence for accepting the null hypothesis; $\beta_{1}=0.5$. The table reports that when $\lambda=0$ then for all the cases of $\rho=0.2,0.5,0.8$, both GLSAD estimator and the proposed PWGLS estimator perform almost equally in the sense of hypothesis testing. For $\lambda=1$, the $p$-value falls around 0.97 for PWGLS and that is around 0.96 for GLSAD which means that the test is highly non-significant or there is no significance difference between the estimated slope value and the parametric (hypothesized) slope value. For 
PWGLS there is stronger evidence to accept the null hypothesis as compared to GLSAD. In other words, the PWGLS estimates remain closer to the true value as compared to GLSAD estimates. This degree of non-significance increases with the increase of the degree of heteroscedasticity and in almost all the cases the p-value remains low for GLSAD when compared with that of the adaptive PWGLS estimator and same sensitivity of the smoothing parameter can also be found here. This also magnifies the adequacy of the proposed estimator even in testing of hypothesis.

Table 6 shows a different picture of p-values under DGP-I (sample size; 300) as in Table 5. For homoscedastic unit-time varying error term $(\lambda=0)$, there is relatively high difference up to 0.05 between the p-values for the both estimators and PWGLS estimator bears high $p$-values. For $\lambda=1, p$-values increase for the both estimators in large sample and remains to some extent high for PWGLS but for $\lambda=2$ and $\lambda=3$, generally, the $p$-values decline for PWGLS and rise for GLSAD. As it is previously discussed that for small sample PWGLS shows better performance as it does in large samples, generally, a similar behaviour for $p$-values is also observed in this table.

Table 7 and Table 8 show the p-values for testing the slope coefficient under DGP-II where regressors are generated by lognormal distribution and the sample sizes are 150 and 300 . The results follow the same routine as they do in Table 5 and 6.

\section{Conclusions}

In this paper, following the work of Li and Stengos (1994) and Roy (1999), we present an adaptive estimator for panel data model with unit-time varying heteroscedasticity of unknown form by replacing kernel estimator, presented by Li and Stengos (1994), with probability weighted moment estimator. The Monte Carlo study under different data generating processes shows the adequate performance of the proposed estimator over the GLSAD estimator suggested by Li and Stengos (1994), specially for small samples and high degree of heteroscedasticity and while comparing with the tedious manipulations and sensitivity of the smoothing parameter in the GLSAD estimator. When one does not have any information on the degree of heteroscedasticity and the share of the different variances of the error terms in the total variance, which would indeed be the case with real data and from more applied point of view, it might be better to use PWGLS. Moreover, our proposed estimator PWGLS also performs with qualitative attractiveness for the hypothesis tests regarding the coefficients while estimating the model. Although, this performance is not very remarkable as compared to that of GLSAD estimator but yet relatively simple computations make it more desirable to use. 
Table 1: Relative Efficiency of the Slope Coefficient: DGP-I, N = 50, T = 3

\begin{tabular}{|c|c|c|c|c|}
\hline & $\lambda=0$ & $\lambda=1$ & $\lambda=2$ & $\lambda=3$ \\
\hline \multicolumn{5}{|c|}{$\rho=0.2$} \\
\hline $\operatorname{GLSAD}(\mathrm{h}=0.8)$ & 1.0094 & 1.0482 & 1.1180 & 1.2490 \\
\hline GLSAD(h = 1.0) & 1.0082 & 1.0476 & 1.1174 & 1.2514 \\
\hline $\operatorname{GLSAD}(\mathrm{h}=1.2)$ & 1.0080 & 1.0475 & 1.1168 & 1.2440 \\
\hline OLS & 2.1358 & 3.3138 & 4.6565 & 5.3927 \\
\hline \multicolumn{5}{|c|}{$\rho=0.5$} \\
\hline GLSAD(h = 0.8) & 1.0087 & 1.0319 & 1.1083 & 1.2116 \\
\hline GLSAD(h = 1.0) & 1.0084 & 1.0303 & 1.1062 & 1.2144 \\
\hline $\operatorname{GLSAD}(\mathrm{h}=1.2)$ & 1.0078 & 1.0320 & 1.1057 & 1.2068 \\
\hline OLS & 1.5060 & 2.3076 & 2.7616 & 2.6960 \\
\hline \multicolumn{5}{|c|}{$\rho=0.8$} \\
\hline $\operatorname{GLSAD}(\mathrm{h}=0.8)$ & 1.0041 & 1.0329 & 1.0907 & 1.1879 \\
\hline $\operatorname{GLSAD}(\mathrm{h}=1.0)$ & 1.0035 & 1.0314 & 1.0884 & 1.1922 \\
\hline $\operatorname{GLSAD}(\mathrm{h}=1.2)$ & 1.0035 & 1.0309 & 1.0880 & 1.1836 \\
\hline OLS & 1.1010 & 1.3259 & 1.4622 & 1.5463 \\
\hline
\end{tabular}

Table 2: Relative Efficiency of the Slope Coefficient: DGP-I, N = 100, T=3

\begin{tabular}{|l|c|c|c|c|}
\hline & $\lambda=0$ & $\lambda=1$ & $\lambda=2$ & $\lambda=3$ \\
\hline \multicolumn{5}{|c|}{$\rho=0.2$} \\
\hline GLSAD(h=0.8) & 1.0481 & 1.0101 & 1.1168 & 1.2449 \\
\hline GLSAD(h=1.0) & 1.0476 & 1.0070 & 1.1159 & 1.2455 \\
\hline GLSAD(h=1.2) & 1.0475 & 1.0067 & 1.1153 & 1.2400 \\
\hline OLS & 3.5777 & 2.3007 & 5.0926 & 5.9372 \\
\hline \multicolumn{5}{|c|}{$\rho=0.5$} \\
\hline GLSAD(h=0.8) & 1.0077 & 1.0295 & 1.1077 & 1.2071 \\
\hline GLSAD $(\mathrm{h}=1.0)$ & 1.0075 & 1.0280 & 1.056 & 1.2080 \\
\hline GLSAD $(\mathrm{h}=1.2)$ & 1.0068 & 1.0291 & 1.050 & 1.2020 \\
\hline OLS & 1.5007 & 2.2854 & 2.7783 & 2.7163 \\
\hline \multicolumn{5}{|c|}{$\rho=0.8$} \\
\hline GLSAD(h=0.8) & 1.0036 & 1.0298 & 1.0890 & 1.1788 \\
\hline GLSAD(h=1.0) & 1.0029 & 1.0287 & 1.0863 & 1.1805 \\
\hline GLSAD(h $=1.2)$ & 1.0024 & 1.0280 & 1.0858 & 1.1738 \\
\hline OLS & 1.0995 & 1.3143 & 1.4331 & 1.5223 \\
\hline
\end{tabular}


Table 3: Relative Efficiency of the Slope Coefficient: DGP-II, N = 50, T=3

\begin{tabular}{|l|c|c|c|c|}
\hline & $\lambda=0$ & $\lambda=1$ & $\lambda=2$ & $\lambda=3$ \\
\hline \multicolumn{5}{|c|}{$\rho=0.2$} \\
\hline GLSAD(h=0.8) & 1.0078 & 1.0529 & 1.0922 & 1.1986 \\
\hline GLSAD(h=1.0) & 1.0064 & 1.0610 & 1.0910 & 1.998 \\
\hline GLSAD(h=1.2) & 0.9975 & 1.0558 & 1.1013 & 1.2054 \\
\hline OLS & 1.9900 & 3.0817 & 4.2250 & 4.8019 \\
\hline \multicolumn{5}{|c|}{$\rho=0.5$} \\
\hline GLSAD(h=0.8) & 1.0079 & 1.0396 & 1.0770 & 1.1611 \\
\hline GLSAD(h=1.0) & 1.0055 & 1.0474 & 1.0742 & 1.1630 \\
\hline GLSAD(h=1.2) & 0.9954 & 1.0435 & 1.0869 & 1.1693 \\
\hline OLS & 1.3992 & 2.1108 & 2.3968 & 2.3399 \\
\hline \multicolumn{5}{|c|}{$\rho=0.8$} \\
\hline GLSAD(h=0.8) & 1.0079 & 1.0252 & 1.0617 & 1.1434 \\
\hline GLSAD(h=1.0) & 1.0022 & 1.0237 & 1.0588 & 1.1466 \\
\hline GLSAD(h $=1.2)$ & 1.0015 & 1.0234 & 1.0585 & 1.1543 \\
\hline OLS & 1.0662 & 1.2823 & 1.3864 & 1.4503 \\
\hline
\end{tabular}

Table 4: Relative Efficiency of the Slope Coefficient: DGP-II, N=100, T=3

\begin{tabular}{|l|c|c|c|c|}
\hline & $\lambda=0$ & $\lambda=1$ & $\lambda=2$ & $\lambda=3$ \\
\hline \multicolumn{5}{|c|}{$\rho=0.2$} \\
\hline GLSAD(h=0.8) & 1.0073 & 1.0591 & 1.0925 & 1.1992 \\
\hline GLSAD(h=1.0) & 1.0024 & 1.0707 & 1.0897 & 1.1951 \\
\hline GLSAD(h=1.2) & 1.0031 & 1.0632 & 1.1054 & 1.2113 \\
\hline OLS & 2.2910 & 3.5635 & 5.0615 & 5.8107 \\
\hline \multicolumn{5}{|c|}{$\rho=0.5$} \\
\hline GLSAD(h=0.8) & 1.0028 & 1.0423 & 1.0899 & 1.1748 \\
\hline GLSAD $(\mathrm{h}=1.0)$ & 1.0024 & 1.0548 & 1.0864 & 1.1698 \\
\hline GLSAD $(\mathrm{h}=1.2)$ & 1.0021 & 1.0463 & 1.0995 & 1.1886 \\
\hline OLS & 1.4516 & 2.2511 & 2.7595 & 2.7047 \\
\hline \multicolumn{5}{|c|}{$\rho=0.8$} \\
\hline GLSAD(h=0.8) & 1.0018 & 1.0183 & 1.0668 & 1.1339 \\
\hline GLSAD(h=1.0) & 1.0007 & 1.0338 & 1.0619 & 1.1282 \\
\hline GLSAD(h=1.2) & 0.9840 & 1.0231 & 1.0831 & 1.1500 \\
\hline OLS & 1.0728 & 1.2972 & 1.3604 & 1.4514 \\
\hline
\end{tabular}


Table 5: P-Value for testing $\mathrm{H}_{0}: \beta_{1}=0.5$ against $\beta_{1} \neq 0.5$ : DGP-I, $\mathrm{N}=50, \mathrm{~T}=3$

\begin{tabular}{|c|c|c|c|c|}
\hline & $\lambda=0$ & $\lambda=1$ & $\lambda=2$ & $\lambda=3$ \\
\hline \multicolumn{5}{|c|}{$\rho=0.2$} \\
\hline PWGLS & 0.9569 & 0.9783 & 0.9748 & 0.9758 \\
\hline GLSAD(h = 0.8) & 0.9400 & 0.9667 & 0.9645 & 0.9566 \\
\hline GLSAD(h = 1.0) & 0.9362 & 0.9618 & 0.9689 & 0.9612 \\
\hline GLSAD(h = 1.2) & 0.9382 & 0.9689 & 0.9679 & 0.9603 \\
\hline \multicolumn{5}{|c|}{$\rho=0.5$} \\
\hline PWGLS & 0.9602 & 0.9606 & 0.9863 & 0.9758 \\
\hline GLSAD(h = 0.8) & 0.9688 & 0.9533 & 0.9583 & 0.9585 \\
\hline GLSAD(h = 1.0) & 0.9698 & 0.9569 & 0.9521 & 0.9521 \\
\hline $\operatorname{GLSAD}(\mathrm{h}=1.2)$ & 0.9697 & 0.9548 & 0.9511 & 0.9508 \\
\hline \multicolumn{5}{|c|}{$\rho=0.8$} \\
\hline PWGLS & 0.9726 & 0.9731 & 0.9717 & 0.9711 \\
\hline GLSAD(h = 0.8) & 0.9734 & 0.9625 & 0.9548 & 0.9609 \\
\hline $\operatorname{GLSAD}(\mathrm{h}=1.0)$ & 0.9814 & 0.9650 & 0.9437 & 0.9564 \\
\hline $\operatorname{GLSAD}(\mathrm{h}=1.2)$ & 0.9782 & 0.9674 & 0.9420 & 0.9543 \\
\hline
\end{tabular}

Table 6: $\mathrm{P}-$ Value for testing $\mathrm{H}_{0}: \beta_{1}=0.5$ against $\beta_{1} \neq 0.5$ : DGP-I, $\mathrm{N}=100, \mathrm{~T}=3$

\begin{tabular}{|l|c|c|c|c|}
\hline & $\lambda=0$ & $\lambda=1$ & $\lambda=2$ & $\lambda=3$ \\
\hline \multicolumn{4}{|c|}{$\rho=0.2$} \\
\hline PWGLS & 0.9833 & 0.9992 & 0.9906 & 0.9920 \\
\hline GLSAD(h=0.8) & 0.9336 & 0.9899 & 0.9806 & 0.9752 \\
\hline GLSAD(h=1.0) & 0.9341 & 0.9927 & 0.9829 & 0.9775 \\
\hline GLSAD(h=1.2) & 0.9426 & 0.9876 & 0.9778 & 0.9722 \\
\hline \multicolumn{5}{|c|}{$\rho=0.5$} \\
\hline PWGLS & 0.9653 & 0.9767 & 0.9995 & 0.9925 \\
\hline GLSAD $(\mathrm{h}=0.8)$ & 0.9822 & 0.9735 & 0.9606 & 0.9577 \\
\hline GLSAD $(\mathrm{h}=1.0)$ & 0.9830 & 0.9778 & 0.9556 & 0.9531 \\
\hline GLSAD $(\mathrm{h}=1.2)$ & 0.9813 & 0.9727 & 0.9545 & 0.9520 \\
\hline \multicolumn{5}{|c|}{$\rho=0.8$} \\
\hline PWGLS & 0.9675 & 0.9866 & 0.9675 & 0.9604 \\
\hline GLSAD(h=0.8) & 0.9695 & 0.9829 & 0.9543 & 0.9679 \\
\hline GLSAD(h=1.0) & 0.9774 & 0.9820 & 0.9467 & 0.9616 \\
\hline GLSAD(h $=1.2)$ & 0.9746 & 0.9866 & 0.9443 & 0.9588 \\
\hline
\end{tabular}


Table 7: P-Value for testing $\mathrm{H}_{0}: \beta_{1}=0.5$ against $\beta_{1} \neq 0.5$ : DGP-II, $\mathrm{N}=50, \mathrm{~T}=3$

\begin{tabular}{|c|c|c|c|c|}
\hline & $\lambda=0$ & $\lambda=1$ & $\lambda=2$ & $\lambda=3$ \\
\hline \multicolumn{5}{|c|}{$\rho=0.2$} \\
\hline PWGLS & 0.9573 & 0.9457 & 0.9384 & 0.9425 \\
\hline $\operatorname{GLSAD}(\mathrm{h}=0.8)$ & 0.9483 & 0.9359 & 0.9341 & 0.9239 \\
\hline $\operatorname{GLSAD}(\mathrm{h}=1.0)$ & 0.9423 & 0.9417 & 0.9320 & 0.9279 \\
\hline $\operatorname{GLSAD}(\mathrm{h}=1.2)$ & 0.9466 & 0.9365 & 0.9294 & 0.9309 \\
\hline \multicolumn{5}{|c|}{$\rho=0.5$} \\
\hline PWGLS & 0.9334 & 0.9508 & 0.9554 & 0.9441 \\
\hline $\operatorname{GLSAD}(\mathrm{h}=0.8)$ & 0.9294 & 0.9406 & 0.9392 & 0.9070 \\
\hline $\operatorname{GLSAD}(h=1.0)$ & 0.9320 & 0.9443 & 0.9413 & 0.9106 \\
\hline $\operatorname{GLSAD}(h=1.2)$ & 0.9387 & 0.9453 & 0.9454 & 0.9115 \\
\hline \multicolumn{5}{|c|}{$\rho=0.8$} \\
\hline PWGLS & 0.9578 & 0.9618 & 0.9400 & 0.9322 \\
\hline $\operatorname{GLSAD}(\mathrm{h}=0.8)$ & 0.9456 & 0.9488 & 0.9070 & 0.9054 \\
\hline $\operatorname{GLSAD}(\mathrm{h}=1.0)$ & 0.9464 & 0.9530 & 0.9109 & 0.9140 \\
\hline $\operatorname{GLSAD}(\mathrm{h}=1.2)$ & 0.9464 & 0.9583 & 0.9120 & 0.9150 \\
\hline
\end{tabular}

Table 8: $\mathrm{P}-$ Value for testing $\mathrm{H}_{0}: \beta_{1}=0.5$ against $\beta_{1} \neq 0.5$ : DGP-II, $\mathrm{N}=100, \mathrm{~T}=3$

\begin{tabular}{|l|c|c|c|c|}
\hline & $\lambda=0$ & $\lambda=1$ & $\lambda=2$ & $\lambda=3$ \\
\hline \multicolumn{5}{|c|}{$\rho=0.2$} \\
\hline PWGLS & 0.9693 & 0.9878 & 0.9813 & 0.9867 \\
\hline GLSAD $(\mathrm{h}=0.8)$ & 0.9505 & 0.9646 & 0.9767 & 0.9701 \\
\hline GLSAD $(\mathrm{h}=1.0)$ & 0.9472 & 0.9635 & 0.9695 & 0.9728 \\
\hline GLSAD $(\mathrm{h}=1.2)$ & 0.9618 & 0.9742 & 0.9720 & 0.9861 \\
\hline \multicolumn{5}{|c|}{$\rho=0.5$} \\
\hline PWGLS & 0.9589 & 0.9867 & 0.9839 & 0.9792 \\
\hline GLSAD $(\mathrm{h}=0.8)$ & 0.9376 & 0.9654 & 0.9805 & 0.9577 \\
\hline GLSAD $(\mathrm{h}=1.0)$ & 0.9377 & 0.9715 & 0.9823 & 0.9574 \\
\hline GLSAD $(\mathrm{h}=1.2)$ & 0.9382 & 0.9599 & 0.9839 & 0.9576 \\
\hline \multicolumn{5}{|c|}{$\rho=0.8$} \\
\hline PWGLS & 0.9531 & 0.9765 & 0.9721 & 0.9618 \\
\hline GLSAD $(\mathrm{h}=0.8)$ & 0.9503 & 0.9573 & 0.9694 & 0.9448 \\
\hline GLSAD $(\mathrm{h}=1.0)$ & 0.9802 & 0.9561 & 0.9690 & 0.9483 \\
\hline GLSAD $(\mathrm{h}=1.2)$ & 0.9823 & 0.9645 & 0.9714 & 0.9452 \\
\hline
\end{tabular}




\section{References}

1. Baltagi, B. H. (1985). Econometric Analysis of Panel Data. John Wiley and Sons.

2. Baltagi, B. H., M. Griffin (1988). A Generalized Error Component Model with Heteroksedastic Disturbances. International Economic Review, 29: 745-753.

3. Carroll, R. D. (1982). Adapting for Heteroscedasticity in Linear Models. The Annals of Statistics, 10: 1224-1233.

4. Delgado, M. A. (1992). Semiparametric Generalized Least Squares Estimation in the Multivariate Nonlinear Regression Model. Econometric Theory, 8: 203-222.

5. Downton, F. (1966). Linear Estimates with Polynomial Coefficients, Biometrika 53, 129.

6. Greenwood, J. A., Landwehr, J. M., Matalas, N. C., and Wallis, J. R. (1979). Probability Weighted Moments: Definition and Relation to Parameters of Several Distributions Expressible in Inverse Form. Water Resour. Res. 15, 1049-1054.

7. Hidalgo, J. (1992). Adaptive Estimation in Time Series Regression Models with Heteroskedasticity of Unknown Form, Econometric Theory. 8: 161-187.

8. Hsiao, C. (1986). Analysis of Panel Data. Cambridge University Press, New York.

9. $\mathrm{Li}, \mathrm{Q}$. and T. Stengos (1994). Adaptive Estimation in the Panel Data Error Component Model with Heteroskedasticity of Unknown Form. International Economic Review, 35: 981-1000.

10. Mazodier, P. and A. Trognon (1978). Heteroksedasticity and Strati.cation in Error Components Models. Annales de l'Insee, 30-31: 451-482.

11. Randolph, W. C. (1988), A Transformation for Heteroksedastic Error Components Regression Models. Economics Letters, 27: 349-354.

12. Rilstone, P. (1991). Some Monte Carlo Evidence on the Relative Efficiency of Parametric and Semiparametric EGLS Estimators. Journal of Business and Economic Statistics, 9: 179-187.

13. Robinson, P. M. (1987). Asymptotic Efficient Estimation in the Presence of Heteroksedasticity of Unknown Form. Econometrica, 55: 817-891.

14. Roy, N. (1999). Is Adaptive Estimation Useful for Panel Models with Heteroscedasticity in the Unit-Specific Error Component? Some Monte Carlo Evidence. Econometric Working Paper: EWP9913, ISSN 1485-6441. 